\title{
Open Social Student Modeling: Visualizing Student Models with Parallel IntrospectiveViews
}

\author{
I-Han Hsiao ${ }^{1}$, Fedor Bakalov ${ }^{2}$, Peter Brusilovsky ${ }^{1}$ and Birgitta König-Ries ${ }^{2}$ \\ ${ }^{1}$ School of Information Sciences, University of Pittsburgh. 135 N. Bellefield Ave., \\ Pittsburgh, PA 15260, USA \\ ${ }^{2}$ Institute for Computer Science, University of Jena, Ernst-Abbe-Platz 2, 07743 Jena, Germany \\ ${ }^{1}\left\{\right.$ ihh4, peterb\}@pitt.edu; ${ }^{2}\{$ fedor.bakalov, birgitta.koenig-ries\}@uni-jena.de
}

\begin{abstract}
This paper explores a social extension of open student modeling that we call open social student modeling. We present a specific implementation of this approach that uses parallel IntrospectiveViews to visualize models representing student progress with QuizJET parameterized self-assessment questions for Java programming. The interface allows visualizing not only the student's own model, but also displaying parallel views on the models of their peers and the cumulative model of the entire class or group. The system was evaluated in a semester-long classroom study. While the use of the system was non-mandatory, the parallel IntrospectiveViews interface caused an increase in all of the usage parameters in comparison to a regular portal-based access, which allowed the student to achieve a higher success rate in answering the questions. The collected data offer some evidence that a combination of traditional personalized guidance with social guidance was more effective than personalized guidance alone.
\end{abstract}

Keywords: Open User Model, Visualization, Parameterized Self-Assessment, Open Student Model

\section{Introduction}

Engaging students with social learning technologies has become an important trend in modern e-learning. One of the biggest challenges is to provide support in the context of social learning, while at the same time allowing students to feel in control. One popular solution to address the issue of control is the so-called open student modeling, an approach that permits the students to observe and reflect on their progress. In particular, visual approaches for open student modeling were explored to provide students with an easy-to-grasp and holistic view of their progress [1-3]. However, most of the open student modeling research focuses on the representation of an individual student -- ignoring the social aspect of learning. In contrast, several social visualization approaches which were explored in an e-learning context [4] focus mainly on student communication and collaboration rather than on the student's progress. Our work attempts to explore the potential of open student modeling and student progress visualization in the context of modern social e-learning. The goal is to extend the benefits of visualizing the student models from the cognitive aspects to 
the social aspects of students. We investigate using an open social student modeling approach (which offers parallel views of multiple student models) to guide students to the most appropriate learning content. In this paper, we explore a specific implementation of the open social student modeling approach based on IntrospectiveViews [12] visualization. We do so in the context of a semester-long classroom study. In the next section, we provide a short review of the related work on open user modeling and social learning. The system and study design are presented in Section 3. Then we report the evaluation results. Finally, we summarize this work and discuss the future research plan.

\section{Related Work}

There are two main streams of work on open student models. One stream focuses on visualizing the model to support students' self-reflection and planning; the other one encourages students to participate in the modeling process, such as engaging students through negotiation or collaboration on the construction of the model [2]. Representations of the student model vary from displaying high-level summaries (such as skill meters) to complex concept maps or Bayesian networks. A range of benefits of opening the student models to the learners have been reported, such as increasing the learner's awareness of the developing knowledge, difficulties and the learning process, and students' engagement, motivation, and knowledge reflection [13]. Dimitrova et al. [5] explore interactive open learner modeling by engaging learners in negotiating with the system during the modeling process. Chen et al. [6] investigated active open learner models in order to motivate learners to improve their academic performance. Both individual and group open learner models were studied; they both demonstrated an increase in reflection and helpful interactions among teammates. Bull \& Kay [7] described a framework to apply open user models in adaptive learning environments and provided many in-depth examples. In our own work on the QuizGuide system [11] we embedded open learning models into the adaptive link annotation and demonstrated that this arrangement can remarkably increase student motivation to work with non-mandatory educational content.

To support social learning, it is common to see the use of the average values of a group to represent a particular aspect in the model. Open group modeling enables students to compare and understand their own states of learning. Such group models have been used to support the collaboration between learners among the same group, and to foster competition in a group of learners [8]. Vassileva and Sun [8] investigated the community visualization in online communities. They summarized that social visualization allows peer-recognition and provides students with the opportunity to build trust in others and in the group. Bull \& Britland [9] used OLMlets to research the problem of facilitating group collaboration and competition. The results revealed that optionally releasing the models to their peers increased the discussion among students and encourages them to start working sooner. CourseVis [10] is one of the few systems providing graphical visualization for multiple groups of users to teachers and learners. It helps instructors to identify problems early on, and to prevent some of the common problems in distance learning. Therefore, it motivates us 
to further investigate the effectiveness of social visualization techniques in the open student model systems.

\section{QuizJET Meets IntrospectiveViews}

To explore the value of open social student modeling, we extended the educational system QuizJET with an open social student modeling interface based on a modified version of the IntrospectiveViews visualization tools. QuizJET is a system for authoring and delivery of parameterized questions on Java programming language. It generates parameterized questions for assessment and self-assessment of students' knowledge on a broad range of Java topics. The implementation and functionalities of QuizJET were described in detail in [11]. The IntrospectiveViews visualization approach was first proposed for scrutinizing semantically-enriched user interest models in [12]. The interface visualizes user interests as a set of keywords displayed on a circular surface gradually painted in shades between red and blue, where the gradient colors denote different degrees of interest. It also allows grouping the items into circular sectors by type, i.e., the semantic class they belong to (e.g. person, company, country, etc.).

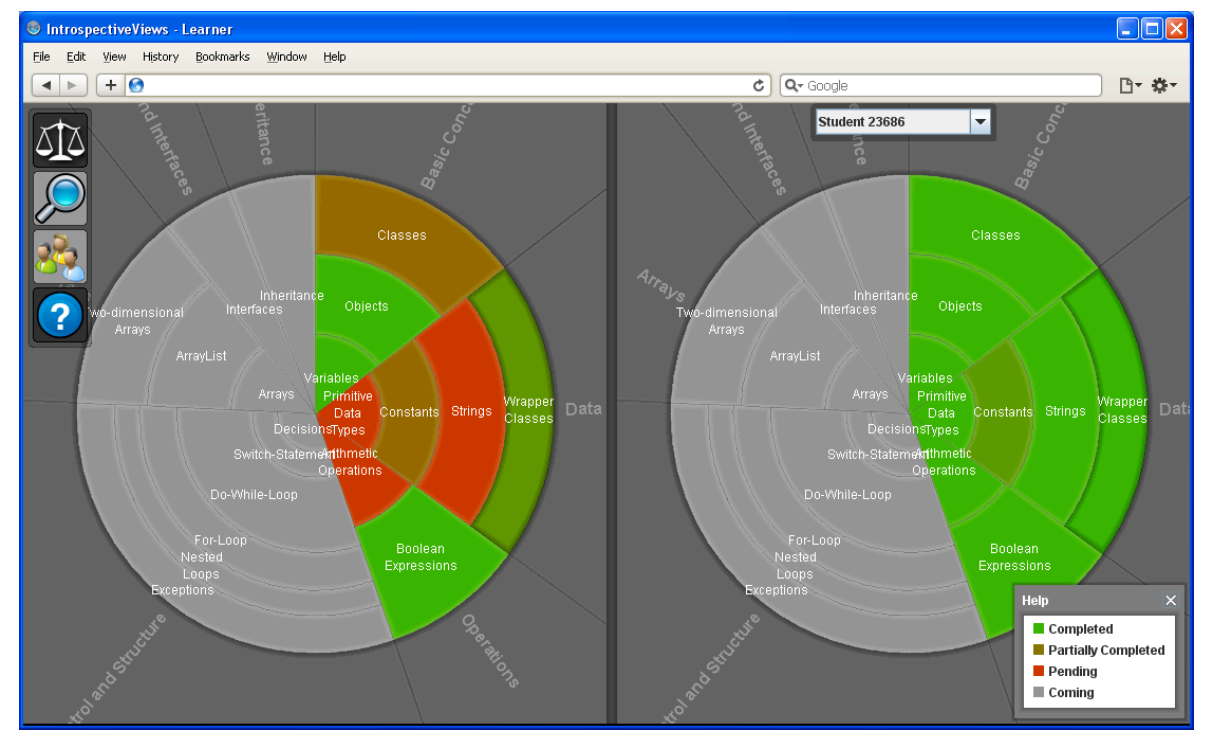

Fig. 1. Parallel IntrospectiveViews. Left pane - visualization of the student's own progress; right pane - visualization of a peer's progress. The circular sectors represent the lectures and the annular sectors represent the topics of individual lectures. The shades of the sectors indicate whether the topic has been covered and for the covered subjects, they denote the progress the student has made. Color screenshots available at: http://www.minervaportals.de/research/introspective-views/.

In [14] we presented an adapted version of IntrospectiveViews, which was 
modified to fit the context of social learning. This version visualizes learner progress rather than user interests and offers parallel views of two student models so that the user can see not only her own model, but also the models of her peers and the class on average. Below, we briefly describe the application of parallel IntrospectiveViews for visualizing student progress on QuizJET questions. For a more detailed description refer to [14].

Figure 1 shows parallel IntrospectiveViews for a student in a class on ObjectOriented Programming (OOP). The visualization consists of two panes: the left pane displays the student's own progress and the right one displays the progress of someone else. Each pane visualizes the respective student's progress as a pie chart. The pie chart representation was chosen because of its capability to visually convey the chronological order of items and their size. The pie chart consists of several circular sectors each representing a class lecture. The lectures are displayed in a clockwise order denoting their pre-requisite sequence, i.e., the order they are taught in class. Lectures may consist of one or several topics, which are represented as annular sectors placed within the circular sector of the corresponding lecture. The radius (width) of annular sectors denotes the amount of readings, quizzes, and exercises assigned to the topic. In a similar way, the span of circular sectors indicates the amount of learning content assigned to the corresponding lecture. Such representation allows the student to easily estimate the amount of work she has to spend on each individual topic or lecture. The shade of each annular sector denotes whether the topic has been covered and, for the covered ones, indicates the progress the student has made with respect to the topic. The sectors painted grey represent the topics that have not been covered yet, whereas the sectors painted a shade from the color range red to green represent the sectors that have been already covered. For the covered topics, the interface displays the student progress. The progress, in the current implementation, is a ratio of successfully completed quizzes to the total quiz count in the topic. If the ratio equals 0 , i.e., no quiz has been successfully completed, the sector is painted red. If it equals 1 , i.e., all quizzes have been completed, the sector appears green. The shades in the range between red and green denote partial completion of the quizzes.

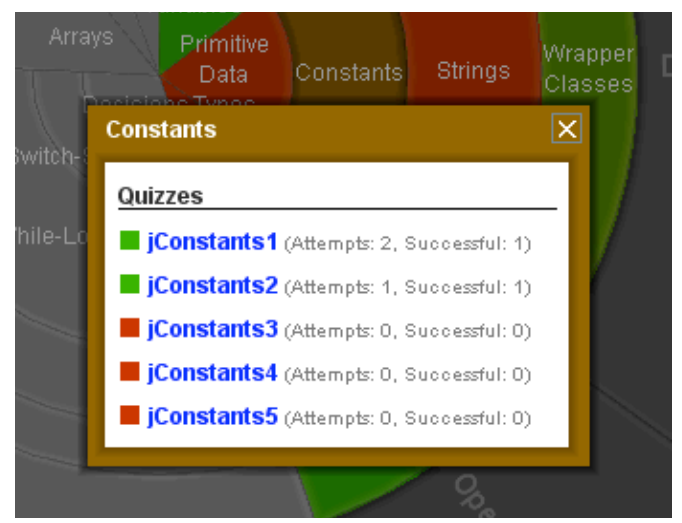

Fig. 2. Quizzes of the selected topic. 
The interface allow the user to see the contents of the corresponding topic by clicking on a particular sector. In the current implementation, the list of questions on the topic is presented when selected (Figure 2). For each question, the interface provides a visual cue indicating the student's progress and displays the total number of attempts the student has made on the quiz and the number of successful attempts. By clicking on a quiz label, the interface will display the quiz in a new window.

Our hypothesis is that such visualization can help the student to plan her class work by providing an overview of her progress in the class and showing the topics that she has already completed as well as those yet to be worked on. In addition to that, we believe that the ability to view someone else's progress can help the student to quickly find the peers that can help with a difficult topic or quiz. The class study described in the next section reveals whether or not this hypothesis is true.

\section{The Classroom Study and the Results}

To assess the impact of our technology, we have conducted a thorough evaluation in a semester-long classroom study. The study was performed in an undergraduate ObjectOriented Programming course offered by the School of Information Sciences, University of Pittsburgh in the Fall semester of 2010. All students received access to self-assessment quizzes through the IntrospectiveViews (IV) interface. The system was introduced to the class at the beginning of the course and served as a nonmandatory course tool over the entire semester. Of the 32 students enrolled in the course, 18 actively used the system. All student activity with the system was recorded. For every student attempt to answer a question, the system stored a timestamp, the user's name, the question, quiz, and session ids, and the results (right or wrong). We also recorded the frequency and timing of student model access and comparisons. Pre- and post- tests were administered at the beginning and the end of the semester in order to measure the gain in students' learning. At the end of the semester, the students were asked to provide their subjective feedback about the system and its features by completing the evaluation questionnaire.

\subsection{Effects on System Usage}

On average, each student attempted 113 different questions and achieved a success rate of $71.35 \%$ on answering the questions. On average, students tried 9 out of 17 distinct topics and 36.5 out of 103 distinct question. The data is summarized in Table 1. Following our prior experience with open student modeling in JavaGuide [11], we expected that the ability to view student knowledge progress would encourage the students to work more with the system. To assess it, we compared the student usage of self-assessment quizzes through IV (Column 1 in Table 1) with the data from a comparable class that accessed quizzes using a traditional course portal with no progress visualization (Column 2 in Table 1) and another class accessing quizzes through an adaptive hypermedia system JavaGuide (Column 3 in Table 1). We found that the social visualization of student models with IntrospectiveViews resulted in a 
$39 \%$ increase in the average attempts compared to the traditional course portal. The students also explored more topics, tried more distinct questions, and accessed the system more frequently. In brief, we observed an increase in all usage parameters similar to that it was observed in a very different JavaGuide interface. At the same time, the increase in usage was not as high as in the case of JavaGuide. As a result, no significant difference on the usage level was found between IV and the portal as well as between IV and JavaGuide.

Table 1. Summary of Basic Statistics of System Usage

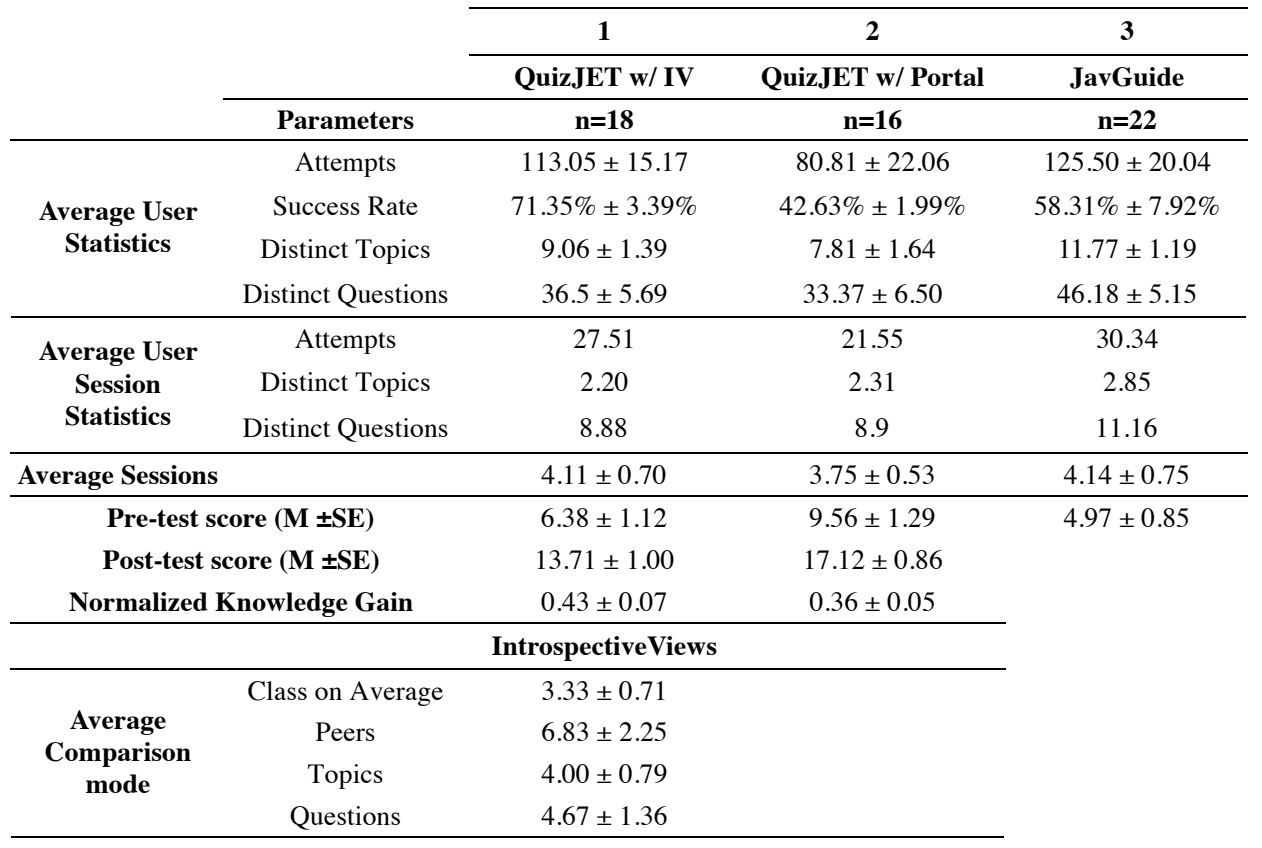

Since the student own knowledge visualization was relatively similar in IV and JavaGuide, a slighter increase in student activity in IV could be attributed to the social side of open social student modeling. While the access to social data could encourage less active users to do more work, it can also discourage very active users from jumping too much ahead of the class. As a result, the difference between the most active and least active users is getting smaller. Evidence that this is really happening is the observed $25 \%$ decrease in standard deviations for the number of attempts. In turn, the class as a whole became a bit less adventurous than in non-social JavaGuide, exploring fewer questions and topics (this is because the variety of topics come to some extent from more active users who run ahead of the class). This effect can be also observed in IV, especially the session level. While the amount of work per session increases for IV, question and topic coverage stays the same.

In sum, as a whole, social guidance provided by the access to class progress mediates the motivating effect of progress visualization by making the whole class a bit less adventurous and more conservative than without social guidance tools. An interesting question is whether a more conservative increase in the amount of work 
and variety of explored context is a good or a bad thing. Our evidence shows that it might actually be a good thing. As Table 1 shows, students using social visualization in IV achieved the highest success rate (a ratio of correct solutions to total attempts) among all conditions. This is significantly higher than for the portal case, $F(1,32)=$ $11.303, p<.01$. The growth of the success rate demonstrates that knowledge-based and social guidance combined are more effective in guiding the students to appropriate questions that they are ready to handle than knowledge-based guidance alone. The community wisdom does matter.

\subsection{The Use of Peer Guidance}

The assumptions about the impact of social features of IV can be validated only if we can show some evidence that these features were really used by students. To collect this evidence, we looked at how students use the provided ability to compare their models with those of their peers' models. We found that students compared their own models to the models of their peers on the average of 6.83 times on average. This is strong evidence that the social features were used and that they had a chance to provide social guidance by affecting student question selection. But can we really argue that peer progress data could guide the student to appropriate topics and questions? Could it be just curiosity? To answer this question, we checked how many times a topic and a question were accessed from the peer model chart rather than from the students' own model of knowledge. We found that on average, students compared to their peers on 4 topics and made 4.67 attempts on the questions initiating from the peers' chart. The final question is whether the guidance obtained by visiting progress data of their peers benefited student learning. We found a correlation between the frequency of peer model comparisons and the learning gain. The more the students compared to their peers, the higher post-quiz scores they received $(\mathrm{r}=0.34 p=0.004)$.

\subsection{Effects on Student Learning}

A study of educational innovation is not complete without the analysis of its impact on student learning. To ensure that the student cohorts were comparable, we first examined the students' pre-test scores. We found no significant differences between groups before using the systems, $F(2,53)=1.644, p=.203, \eta^{2}=.057$. The assumption of homogeneity of variance was met, Brown-Forsythe $F(2,53)=1.644, p=.207$. We found that in both conditions (IV and portal), the students achieved a significant knowledge growth as measured by pre- and post- test scores, $\mathrm{t}_{1}(17)=7.203, \mathrm{p}<.01$; $\mathrm{t}_{2}(15)=6.108, \mathrm{p}<.01$. To compare learning gains under these two conditions, we calculated the Normalized Knowledge Gain (NKG) based on formula (1). While the average NKG was slightly higher in the IV group, we did not find significant differences between these two conditions. It should be noted, however, that our experiment was performed in a non-controlled classroom context where the systems were used as just supplementary course tools. The students were able to learn the subject by many ways with the QuizJET/Portal system being just one of many factors which may have contributed to the learning. 


$$
N K G=\frac{\text { posttest }- \text { pretest }}{\text { max score }- \text { pretest }}
$$

Table 2. Questionnaire

Usefulness

A.1. The interface helps me to understand how the class content is organized.

A.2. The interface helps me to identify my weak points.

A.3. The interface helps me to plan my class work.

A.4. The interface helps me to access quizzes.

A.5. The comparison mode of the interface motivates me to progress on the quizzes.

A.6. The comparison mode of the interface helps me to find the classmates who can help on difficult topics.

Ease of Use

B.1. The interface is easy to use.

B.2. The interface is user friendly.

B.3. The interface requires the fewest steps possible to accomplish what I want to do with it.

Ease of Learning

C.1. I learned how to use the interface quickly.

C.2. I easily remember how to use the interface.

C.3. It is easy to learn how to use the interface.

\section{Satisfaction}

D.1. I am satisfied with the interface.

D.2. The interface is fun to use.

D.3. The interface is pleasant to use.

D.4. I would recommend the interface to my classmates.

Privacy and Data Sharing

E.1. I like the idea of comparing my progress with other students.

E.2. I feel comfortable sharing my progress with others.

E.3. I do not mind that my progress is displayed anonymously in the average progress of the entire class.

E.4. I would like to view progress of other students because:

1. $\mathrm{S} / \mathrm{He}$ and I are friends

2. I know $\mathrm{s} /$ he is a good student

3. I know s/he is good at specific topic

4. I am just curious

5. Other

E.5. I am willing to share:

1. My overall progress with: no one / selected classmates / everyone

2. My good progress topics with: no one / selected classmates / everyone

3. My overall success rate with: no one / selected classmates / everyone

4. My good success rate topics with: no one / selected classmates / everyone.

5. Selected topics with: no one / selected classmates / everyone.

6. Other 


\subsection{Subjective Evaluation Results}

Out of the 18 IV users, 13 completed the questionnaire. For the purpose of analysis, we classified 17 questions into 5 categories (Table 2). From the usefulness perspective, $84.5 \%$ of the students strongly agreed or agreed that the clockwise piechart design helped them to understand how the class content is organized. $76.9 \%$ of the students agreed or strongly agreed that the interface helped them to identify their weak points. $84.6 \%$ of the students agreed that the interface helped them to access the quizzes. $61.5 \%$ of the students agreed that the comparison mode motivated them to progress on the quizzes. However, there were $76.9 \%$ of students who did not think the comparison mode allowed them to identify a classmate to help them on difficult topic regardless of the positive effects of using the comparison mode (proven in the previous section). The results suggested that the students generally had a high opinion of agreement on the usefulness of the system and indentified the system's inability to find a comparable peer from the current design. Considering the Ease of Use \& Ease of Learning in the system, students found it easy to learn how to use the system (92.3\%), easy to remember how to use it $(92.3 \%)$ and learned how to use it quickly $(84.6 \%)$. They considered that the interface was easy to use $(76.9 \%)$, it was user friendly (69.2\%) and required fewest steps to accomplish the task ${ }^{1}(66.7 \%)$. There was not a single strong disagreement with the questions of this category. In the category of Satisfaction, students liked the system. $76.9 \%$ were strongly satisfied with the system. They determined that the interface was fun $(69.2 \%)$ and pleasant $(76.9 \%)$ to use. $91.3 \%$ of the students would recommend it to their classmates. In terms of Privacy and Data Sharing, $84.6 \%$ of the students appreciated the feature of comparing their progress with others. $69.2 \%$ of them felt comfortable in sharing their progress with others. However, some of them had concerns on sharing the data with others. $15.4 \%$ of them do not want to share any data with others at all. $30.8 \%$ of them would like to selectively share data with others, for example, display the model anonymously or selectively share the data (either their progress or success). We also investigated the reasons of why students view the progress of other students. We found that $46.2 \%$ of the students viewed others progress out of curiosity. $46.2 \%$ of them knew the ones they viewed are good students or are good at specific topic. To extend the current model on aspects other than progress, we also collected students' opinions on such attributes as success rate, selected topics, good progress and good success rate. $46.2 \%$ of the students are willing to share everything to everyone. $23.1 \%$ are willing to share their overall progress to selected people. $23.1 \%$ of them would only share the good progress or success rate to everyone. Only 1 student $(7.6 \%)$ was extremely private and was not willing to share anything to anyone. The results indicated that students were generally positive toward the data sharing idea provided the privacy management to make them feel in charge. Figure 3 shows the detail percentages for each question.

\footnotetext{
${ }^{1}$ One of the survey participants did not answer this question (B.3). The percentile was calculated based on the responses from the remainder of the participants.
} 


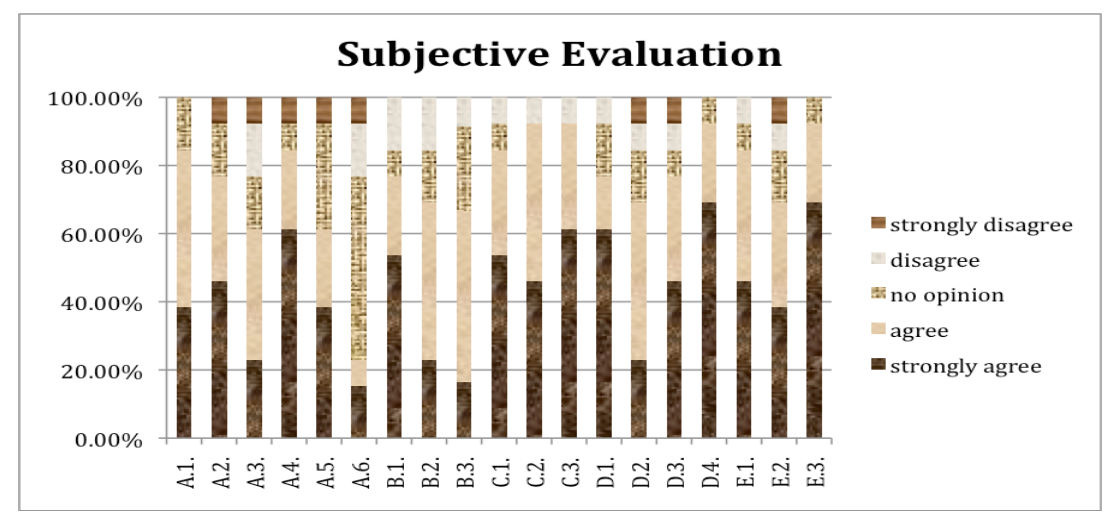

Fig. 3. Subjective evaluation results.

\section{Summary \& Future Work}

In this paper, we presented a specific implementation of the open social student modeling approach based on the parallel IntrospectiveViews interface. This interface was used to provide access to QuizJET parameterized self-assessment questions in an introductory programming class. The parallel IntrospectiveViews interface allowed the students to visualize not only the student's own model, but also to display parallel views of the models of their peers and the cumulative model of the entire class. The system was evaluated in a semester-long classroom study. While the use of the system was non-mandatory, it has been used very actively. Moreover, the social features provided by the interface were used for both progress comparison and navigation. We observed that the parallel IntrospectiveViews interface caused an increase in all the usage parameters in comparison to a regular portal-based access system. While the increase was slightly smaller and conservative in comparison to the similar increase caused by our earlier system (JavaGuide) non-social open student modeling interface of our earlier system JavaGuide, the IntrospectiveViews interface allowed the student to achieve a higher success rate in answering the questions. In addition, the system and most of its features were highly praised by the students.

Our current results are encouraging and suggest new challenges for the future work. Based on our experience, we identified five areas for improvement in the future.

(1) Adaptive navigation support: based on our previous experiences [11] adaptive navigation support can dramatically increase the likelihood of answering the questions correctly. Therefore, the current design can be further improved with the additions of adaptive navigation support feature such as providing icon abstractions etc.

(2) Personalized guidance: the positive correlation between comparison with peers and learning gain encourages us to further look at the effects of comparison between students with different levels of knowledge; for example, a recommendation about whose models to explore. 
(3) Privacy management: students have different levels of concerns about the privacy side for data sharing. Therefore, in the future, we have to enable the privacy setting in a sensitive manner to accommodate assorted scenarios.

(4) Visualizing models of multiple peers: to help users to navigate through the peers' models, the interface should be able to display multiple models at a time. The next version will contain a pane listing miniature copies of progress pie charts of all classmates. The user will be able to sort peers by overall progress, progress in a given topic, name, and other attributes.

(5) Collaboration features: in order to facilitate collaboration among students, we plan to add a feature for sending messages from the interface and a feature allowing students to set the status indicating their willingness to help.

\section{Acknowledgment}

This research was supported in part by the National Science Foundation under Grant No. 0447083

The development of IntrospectiveViews was carried out in the Minerva ContextAdaptive Portals project funded by the IBM Deutschland Research \& Development $\mathrm{GmbH}$.

\section{References}

1. Bull, S. (2004) Supporting learning with open learner models. In: Proceedings of 4th Hellenic Conference on Information and Communication Technologies in Education, Athens, Greece, September 29 - October 3, 2004, pp. 47-61

2. Mitrovic, A., Martin, B.: Evaluating the Effect of Open Student Models on SelfAssessment. Int. J. of Artificial Intelligence in Education 17(2), 121-144 (2007)

3. Zapata-Rivera, J.D., Greer, J.E., (2004). Visualizing and inspecting Bayesian belief models. In: International Journal of Artificial Intelligence in Education IJCAI, 14, pp. 1-37. IOS Press.

4. Vassileva, J. Toward Social Learning Environments. IEEE Transactions on Learning Technologies, 2008, 1(4), p. 199-214.

5. Dimitrova, V., Self, J., Brna, P . (2001) Applying Interactive Open Learner Models to Learning Technical Terminology. Proceedings of User Modeling'2001, Springer, pp. 148-157.

6. Chen, Z.H., Chou, C.Y., Deng, Y.C., and Chan, T.W. (2007). Active Open Learner Models as Animal Companions: Motivating Children to Learn through Interaction with My-Pet and Our-Pet, International Journal of Artificial Intelligence in Education, 17(3): 217-226

7. Bull, S. \& Kay, J. (2007). Student Models that Invite the Learner In: The SMILI Open Learner Modelling Framework, International Journal of Artificial Intelligence in Education 17(2), 89-120. 
8. Vassileva J., Sun L. (2007) Using Community Visualization to Stimulate Participation in Online Communities.e-Service Journal, 6 (1), 3-40.

9. Bull, S. \& Britland, M. (2007). Group Interaction Prompted by a Simple Assessed Open Learner Model that can be Optionally Released to Peers, in P. Brusilovsky, M. Grigoriadou \& K. Papanikolaou (eds), Proceedings of Workshop on Personalisation in E-Learning Environments at Individual and Group Level, User Modeling 2007.

10. Mazza, R. \& Dimitrova, V. (2007). CourseVis: a graphical student monitoring tool for supporting instructors in web-based distance courses. International Journal of Human-Computer Studies, 65, 2, 125-139.

11. Hsiao, I., Sosnovsky,S. and Brusilovsky, P. (2010) Guiding Students to the Right Questions: Adaptive Navigation Support in an E-learning System for Java Programming, Journal of Computer Assisted Learning, Volume 26 Issue 4, Pages $270-283$.

12. Bakalov, F., König-Ries, B., Nauerz, A., and Welsch, M. IntrospectiveViews: an Interface for Scrutinizing Semantic User Models. In Proc. of the 18th Intl. Conf on User Modeling, Adaptation and Personalization (2010).

13. Bakalov, F., König-Ries, B., Nauerz, A., and Welsch, M. Scrutinizing User Interest Models with IntrospectiveViews. In Adjunct Proc. of the 18th Intl. Conf on User Modeling, Adaptation and Personalization (2010).

14. Bakalov, F., Hsiao, I., Brusilovsky, P., and König-Ries, B. Visualizing Student Models for Social Learning with Parallel IntrospectiveViews. In Proc. of the Workshop on Visual Interfaces to the Social and Semantic Web at the International Conference on Intelligent User Interfaces (2011).

15. Vassileva, J.: Open Group Learner Modeling, Interaction Analysis and Social Visualization. In: Dimitrova, V., Tzagarakis, M., Vassileva, J. (eds.) Proceedings of Workshop on Adaptation and Personalisation in Social Systems: Groups, Teams, Communities. Held in conjunction with UM 2007, (2007) 